\title{
A survey of intestinal parasitic infections among Dega Ochollo primary school children, Ochollo, South Ethiopia
}

\author{
Gessessew Bugssa ${ }^{1,}$, , Berhe Dessalegn ${ }^{2}$, Megbaru Alemu ${ }^{1}$, Haile Desta ${ }^{1}$, Tensay Kahsay $^{3}$ \\ ${ }^{1}$ Institute of Biomedical Sciences, College of Health Sciences, Mekelle University, Mekelle, Ethiopia \\ ${ }^{2}$ Department of Public Health College of Health Sciences, Adigrat University, Adigrat, Ethiopia \\ ${ }^{3}$ Mizan Tepi University, College of Health Sciences, Department of Nursing, Mizan, Ethiopia \\ Email address: \\ hamuge123@gmail.com (G. Bugssa),berhe.desg@gmail.com (B. Dessalegn), megibgesep@gmail.com (M. Alemu), \\ haile.desg@gmail.com (H. Desta), tensay.gkahsay@gmail.com (T. Kahsay)
}

\section{To cite this article:}

Gessessew Bugssa, Berhe Dessalegn, Megbaru Alemu, Haile Desta, Tensay Kahsay. A Survey of Intestinal Parasitic Infections among Dega Ochollo Primary School Children, Ochollo, South Ethiopia. Science Journal of Public Health. Vol. 3, No. 1, 2015, pp. 56-60.

doi: $10.11648 /$ j.sjph.20150301.20

\begin{abstract}
Background: Intestinal parasitic infections remain an important cause of morbidity and mortality in developing countries especially among pediatric. Objective: To determine the prevalence of S.mansoni and soil transmitted helminthes among Ochollo primary school students, Ochollo, southwest Ethiopia. Methods: A cross-sectional study design was employed to assess the prevalence of S.mansoni and soil transmitted helminthes in the study area. The study was conducted between December 2011 and April 2012 in Dega Ochollo complete primary school. A convenient sampling method was employed and a total of 600 students were sampled for the study. Sociodemographic information data such as age, sex, place of birth, educational level and other information was collected from the study participants. Besides, stool specimen was collected from each school children for parasitological examination. Normal saline and Formol ether concentration techniques were used to examine the stool specimen. Data were entered using Epi Info version 3.0 and exported to SPSS version16.0 software package for statistical analysis. Result: Sociodemographic data showed that out of the 600 students sampled, 523 students had participated in the study. Of which, 272 were males and 251 were females. The mean age for the students was recorded to be $11.36 \pm 2.96$ years. Microscopic stool examination showed an overall prevalence of intestinal parasites to be $56.8 \%$. Out of the positive cases, $148(49.8 \%)$ were males; whereas $149(50.2 \%)$ were females. It was also found that $139(26.57 \%)$ of students which belonged to the age group 10 years old or less were positive for at least one intestinal parasite. Among the intestinal helminthes, A.lumbricoides was the predominant parasite (42.1\%) followed by T.trichuria (31.0\%), Hook worm (3.6\%) and H.nana $(1.0 \%)$, respectively. According to the findings of the study, the prevalence of A.lumbricoides and T.trihuria was higher among the female study subjects than their counter parts. Single, double, triple as well as quadriple infections with intestinal parasites were also found among the school children. Based on the study, majority (37.28\%) of the study population had single infection; whereas $19.5 \%$ of the cases had multiple infections.Conclusion: Intestinal parasites were prevalent in varying magnitude among schoolchildren found in Ochollo village.This calls for the institution of control measures including treatment of infected individuals, improvement of sanitation and provision of clean water, and health education.
\end{abstract}

Keywords: Survey, S. Mansoni, Intestinal Parasites, Children, Ochollo, South Ethiopia

\section{Introduction}

Intestinal parasites and protozoan infections are amongst the most common infections worldwide. It is estimated that some 3.5 billion people are affected, and that 450 million are ill as result of these infections, the majority being children (1).

The soil-transmitted helminthes including Ascaris (A.) lumbricoides, hookworm and Trichuris (T.) trichuria are the most prevalent infecting an estimated one-sixth of the global population (2). Intestinal parasitic infections remain an important cause of morbidity and mortality in developing countries especially among pediatric (3). According to WHO (1997) estimation, globally there are 800-1,000 million cases of ascariasis, 700-900 million cases of trichiuriasis, 200 
million cases of giardiasis, and 500 million cases of amoebiasis (4). However, recent estimates suggest that A.lumbricoides can infect over a billion, T.trichiura 795 million, and hookworms 740 million people (5). Enterobius (E.)vermicularisis also among the most prevalent parasite infecting up to one third of children worldwide (6). Schistosomiasis also remains one of the most prevalent parasitic diseases in the world. It is endemic in 76 countries and continues to be public health concern in developing countries. Approximately, $80 \%$ of the 200 million people infected worldwide live in sub-Saharan Africa where Schistosoma(S.) mansoni and S.haematobium are widespread (7).

Infection with intestinal helminthes has been associated with stunting of linear growth, physical weakness and low educational achievement in schoolchildren (8). Furthermore, chronic intestinal parasitism has become the subject of speculation and investigation in relation to the spread and severity of tuberculosis, malaria and other infectious diseases of viral origin (9). Children are likely to be more active in the infected environment by parasites and often employ unhygienic behaviors. Most of the time these potential carriers are crowded together for long periods of time in schools, orphanages and slums which increase the probability of transmission or environmental contamination with the parasites (5).

In Ethiopia, intestinal schistosomiasis and soil transmitted helminthiais are the major causes of mortality and morbidity $(8,9)$. A considerable proportion of annual visits at outpatient services of the health institutions are due to such infections (10). Several studies also indicated that the prevalence of parasitic infections is high in different parts of the country $(8$, 11-15).

Studying the prevalence various intestinal parasitic infection is a prerequisite not only for formulation of appropriate control strategies but also to predict risk for communities under consideration. So far, no studies have been conducted on the prevalence of S.mansoni and soil transmitted helminthes in Ochollo, South Ethiopia. Therefore, this study will have paramount importance in high lighting the distribution of these diseases and addresses the gaps in information about the status of these parasites in the study area.

\section{Methods and Materials}

\subsection{Study Design}

A cross-sectional study design was employed to assess the prevalence of S.mansoni and soil transmitted helminthes in the study area.

\subsection{Study Area and Period}

The study was conducted between December 2011 and April 2012 in DegaOchollo complete primary school. The school is found in a village called Ochollo which is located in the south west Ethiopia, in the Gamogofa administrative region in Arbaminch Zuria Wereda, lying half way between Chencha and Arbaminch. Ochollo farmers' association, with a population of around 5000 clustered densely on the top of a hill, is located on the Rift Valley above the west shore of Lake Abaya at $60^{\circ} 11^{\prime} \mathrm{N}$ and $37^{\circ} 41^{\prime} \mathrm{E}$ 9. The area covers approximately 25 square kilometers and is divided in to four sub-kebelesnamely: Domma, Gucha, Keya and Zuza. The altitude of the study area ranges between 2000 and $2200 \mathrm{~m}$ above sea level. DegaOchollo complete primary school is the only school in that village.

\subsection{Source Population}

The source of populations for this study was all students of DegaOchollo complete primary school who meet the inclusion criteria.

\subsubsection{Inclusion Criteria}

Students of DegaOchollo who are volunteers were eligible to participate in the study.

\subsubsection{Exclusion Criteria}

Students with mental problem and non-volunteers were excluded from the group.

\subsection{Sampling Technique and Sample Size}

A convenient sampling method was employed. There were 600 students in DegaOchollo complete primary school. For ethical reasons, all students attending school in that academic year were sampled for the study. Hence, all the students of the school which were voluntary to participate in the study were examined for the presence of intestinal parasites.

\subsection{Sociodemographic Data}

Sociodemographic information data such as age, sex, place of birth, educational level and other information was collected from the study participants. Besides, stool specimen was collected from each school children for parasitological examination.

\subsection{Parasitological Examination}

After collecting sociodemographic data, the students were then supplied with labeled stool containers with tight covers bearing serial numbers of the subjects and were asked to put about five grams of stool. The entire stool samples were received at an organized central place and a small portion of the specimens were processed using direct saline wet mount for motile intestinal parasites just immediately after delivery of the stool. The rest portion of the stool samples were preserved in $10 \%$ formalin for formol ether concentration technique and were transported to Addis Ababa University, Medical faculty, department of Microbiology, Immunology and Parasitology laboratory.

\subsection{Normal Saline (Wet Mount)}

The stool samples were examined macroscopically for the presence of any intestinal parasites, for consistency and for 
any other physical abnormalities. The samples were then emulsified with 3-4 $\mathrm{ml}$ of normal saline, then a drop of emulsified portion were placed on a glass slide and covered with cover slip. Then microscopic examination was made for presence of any trophozoites, larva, ova and cyst under 10x and 40x objective lens (16).

\subsection{Formol-Ether Concentration Technique}

The stool samples were concentrated and mixed well after arrival at the Medical Faculty, Addis Ababa University. Two grams of each stool sample was emulsified with $3-4 \mathrm{ml}$ of $10 \%$ formal saline, mixed thoroughly and passed through gauze. Four $\mathrm{ml}$ of diethyl ether was added and mixed by inverting and intermittent shaking for 1 minute and centrifuged at $3,000 \mathrm{rpm}$ (revolution per minute) for 5 minutes. After centrifugation, the supernatant (the layer of ether, debris and formal saline) was discarded and the sediment (containing the parasite at the bottom of the test tube) was re-suspended in formal saline. Finally, the smear was prepared from the sediment and observed under light microscope with 10x and 40x objectives for the presence of ova, cyst and larval stage of intestinal parasites (17).

\subsection{Ethical Clearance}

Ethical clearance and approval of the study protocol was obtained from Addis Ababa University, Institutional Review Board (IRB) of the Aklilu Lemma Institute of Pathobiology. Further permission was obtained from the Ochollo Farmers' Association and DegaOchollo primary school. Then written consent was observed from principal of the school and oral consent was obtained from the study participants. Furthermore, the risks involved in the process and the benefits that would be obtained from the study were explained briefly to both the teachers as well as the students. The participants were assured that their name would not be used and all the information would be at utmost confidential. Subjects which were found positive were provided with appropriate anti parasitic drugs with the recommended doses.

\subsection{Data Analysis}

Data were entered using Epi Info version 3.0 and exported to SPSS version16.0 software package for statistical analysis. Data were presented using tables, graph and descriptive statistics.

\section{Results}

\subsection{Sociodemographic Data}

Out of the 600 students sampled for the study, 523 had participated in the current survey. Of which, 272 were males and 251 were females. The age distribution of the students showed that $239(45.7 \%)$ students were 10 years old or less, $245(46.85 \%)$ ranged from 11 to 15 years and 39 students $(7.47 \%)$ were 16 years of age and above. The mean age for the students was recorded to be $11.36 \pm 2.96$ years ranging from 6 to 25 years (Table 1). Of the study participants, $356(68.1 \%)$ of them were Orthodox and $167(31.9 \%)$ were protestant by religion.

Table 1. Age and Sex distribution of Dega Ochollo primary school students, Ochollo, south Ethiopia.

\begin{tabular}{llll}
\hline $\begin{array}{l}\text { Age group (in } \\
\text { Years) }\end{array}$ & Sex & \multicolumn{2}{l}{ Total } \\
\cline { 2 - 3 } & Male & Female & \\
\hline $6-10$ & $133(25.43 \%)$ & $106(20.28 \%)$ & $239(45.71 \%)$ \\
$11-15$ & $115(21.99 \%)$ & $130(24.86 \%)$ & $245(46.85 \%)$ \\
$>=16$ & $24(4.59 \%)$ & $15(2.87 \%)$ & $39(7.46 \%)$ \\
Total & $272(52.01 \%)$ & $251(47.830 \%)$ & $523(100.00)$ \\
\hline
\end{tabular}

The overall prevalence of intestinal parasitic infections in the examined children was $56.8 \%(297 / 523)$. Out of these positives, $49.8 \%$ were males $(n=148)$ and $50.2 \%$ were females $(n=149)$. According to the age groups, the prevalence of intestinal helminthes infections in children having 6-10,11-14 and > 16 years old was $26.57 \%(139 / 239)$; $26.77 \%$ (140/245) and 3.45 (18/39), respectively (Table 2).

Microscopic examination of stool samples showed that among the 523 school children examined for the presence of parasites, the overall prevalence of intestinal parasites was found to be $56.8 \%$. Out of these positive cases, $148(49.8 \%)$ were males whereas $149(50.2 \%)$ were females.

We also assessed the prevalence of intestinal helminthes among the different age groups of the study subjects. Accordingly, it was found that $139(26.57 \%)$ of students which belonged to the age group 10 years old or less were positive for at least one intestinal helminthic parasite whereas $140(26.77 \%)$ of students lying in the age group 11 to 15 years were also positive for at least one parasite.

Table 2. The distribution of intestinal parasites by age in DegaOchollo primary school students, Ochollo, South Ethiopia.

\begin{tabular}{llll}
\hline \multirow{2}{*}{ Age Group } & \multicolumn{2}{l}{ Presence of intestinal parasite } & \multirow{2}{*}{ Total } \\
\cline { 2 - 3 } & Positive & Negative & \\
\hline $6-10$ & $139(26.57 \%)$ & $100(19.12 \%)$ & $239(45.69)$ \\
$11-15$ & $140(26.77 \%)$ & $105(20.08 \%)$ & $245(46.85 \%)$ \\
$>=16$ & $18(3.45 \%)$ & $21(4.02 \%)$ & $39(7.47 \%)$ \\
Total & $297(56.79 \%)$ & $226(43.21 \%)$ & $523(100 \%)$ \\
\hline
\end{tabular}

The prevalence of each parasite was also assessed among the children of DegaOchollo primary school. Among the intestinal helminthes, A.lumbricoides was the predominant parasite $(42.1 \%)$ followed by T.trichuria (31.0\%), Hook worm (3.6\%) and H.nana (1.0\%), respectively. According to the findings of the study, the prevalence of A.lumbricoides and T.trichuria was higher among the female study subjects than their counter parts. However, the difference was not statistically significant. Inaddition to intestinal helminthes, intestinal protozoan parasites were also detcted among the study subjects (Table 3 ). 
Table 3. The prevalence of different intestinal parasitic infections by age andsex among DegaOchollo primary school students, Ochollo, South Ethiopia.

\begin{tabular}{lllll}
\hline \multirow{2}{*}{ Type of parasite } & Sex & \multicolumn{3}{l}{ Overall prevalence } \\
\cline { 2 - 5 } & Male & Female & Frequency & Percent (\%) \\
\hline A.lumbricoides & $109(49.5 \%)$ & $111(50.5 \%)$ & $220(523)$ & 42.1 \\
T.trichuria & $72(44.4 \%)$ & $90(55.6 \%)$ & $162(523)$ & 31.0 \\
Hook worm & $10(52.6 \%)$ & $9(47.4 \%)$ & $19(523)$ & 3.6 \\
E.histolytica/dispar cyst & $6(60.0 \%)$ & $4(40.0 \%)$ & $10(523)$ & 1.9 \\
H.nana & $2(40.0 \%)$ & $3(60.0 \%)$ & $5(523)$ & 1.0 \\
S.mansoni & $4(100.00)$ & $0(0.0 \%)$ & $4(523)$ & 0.8 \\
S.stercoralis larvae & $2(50.0 \%)$ & $2(50.0 \%)$ & $4(523)$ & 0.8 \\
E.vermicularis & $1(50.0 \%)$ & $1(50.0 \%)$ & $2(523)$ & 0.4 \\
G.lamblia cyst & $1(100.0 \%$ & $0(0.0 \%)$ & $1(523)$ & 0.2 \\
\hline
\end{tabular}

Single, double, triple as well as quadriple infections with intestinal parasites were also found among the school children. The majority of the study population $(37.28 \%)$ had single infection whereas $19.5 \%$ of the cases had multiple infections i.e. more than two parasites (Figure 1). There was no significant association between multiple infections and sex and age.

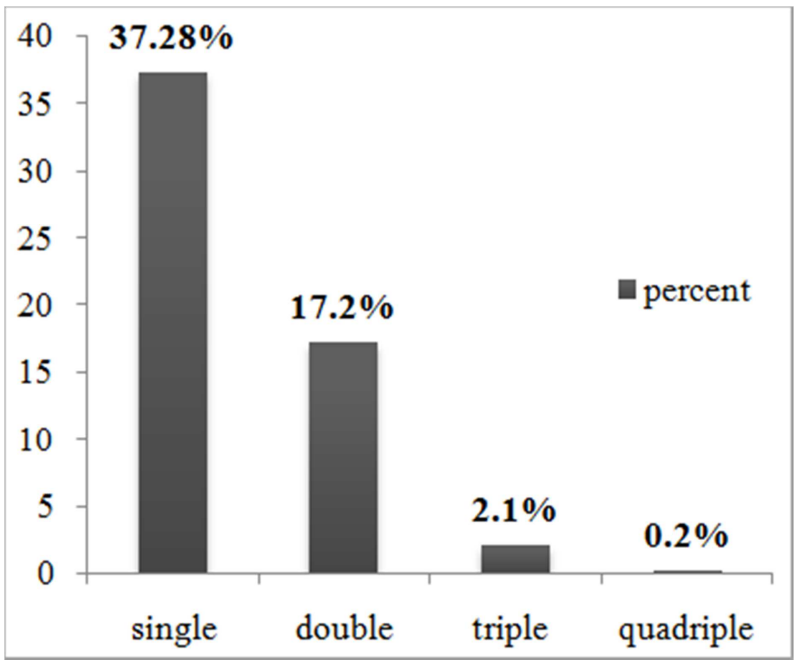

Figure 1. The Prevalence of single and mixed intestinal parasitic infections among DegaOchollo primary school students, Ochollo, South Ethiopia.

\section{Discussion}

A study on the prevalence of intestinal parasites in different districts is a principal objective to identify high-risk communities and devise appropriate intervention (18). We attempted to assess the prevalence of different intestinal helminthic infections in school children in Ochollo district, South Ethiopia. Our findings demonstrate the occurrence of several intestinal helminthes among the schoolchildren which has public health significance.

The finding of this study revealed that various intestinal helminthes and protozoan parasites were common in students of DegaOchollo primary school. Although there are no previous studies conducted in the area, these findings are more or less similar to other studies conducted on other parts of the country. A study conducted by Dejene et al. in Waja primary school, northern Ethiopia, showed an overall prevalence of intestinal parasitic infection of $62.5 \%$ (12). Our findings were, however, higher than those reported by other researchers $(10,15)$ who reported a prevalence rate of $28.6 \%$ and $27.2 \%$, respectively in two different localities at eastern Ethiopia.

However, the finding of our study was higher compared to the findings from Tigray and Babile town (eastern Ethiopia) revealed overall prevalence of $28.6 \%$ and $27.2 \%$, respectively $(10,15)$.

Higher rate $(42.1 \%)$ of A.lumbricoides was observed in this study. This finding agrees with a study conducted in children in Chilga district, North West Ethiopia where the prevalence was found to be $42.9 \%$ (19). Another study conducted in Zarima showed lower prevalence $(21.9 \%)$ of A.lumbricoides(20).This discrepancy could be due to altitudinal difference between the study areas. The other intestinal helminthic parasite which was dominant in the school children was T.trichuria with prevalence rate of $14.8 \%$. This was by far higher than a study conducted in Zarima, Amhara Region (20).The differences in prevalence among the different communities appear to be associated with environmental sanitation, water supply and socioeconomic status of households, although this needs to be verified in more extensive follow up studies. However, infection from hookworms was found to be lower (3.6\%). This report contradicts with the findings conducted in Adarkay district where prevalence of infection was found to be $20.2 \%$ (21).However the prevalence of S.mansoni in the study area was $0.8 \%$ which is lower compared to studies conducted elsewhere in the country $(8,12,15,20,22)$. These differences in the prevalence of S.mansoni may be attributed to altitude variations, availability of streams/rivers, ponds and playing habits of school children.

Moreover, the current report also revealed that hook worm infection was higher in boys $(52.6 \%)$ compared to girls $(47.4 \%)$. This goes in agreement with previous study conducted in southwest Ethiopia where higher prevalence of hook worm was recorded for boys (14). However, our finding contradicts with other studies where hook worm infection was higher lower among girls $(15,23)$. 
Furthermore, the findings of the current study also depicted that intestinal helminthic infections are more prevalent in the age groups 15 years or less. This may signify that younger children are more exposed since they habitually play in the open fields (13) and commonly involve themselves fully in activities that bring them in contact with the source of infection (24).

\section{Conclusion and Recommendations}

Our findings confirm the occurrence of several intestinal parasitic infections in varying magnitude among schoolchildren in Ochollo village, South Ethiopia. This calls for the institution of control measures including treatment of infected individuals, improvement of sanitation and provision of clean water, and health education.

\section{References}

[1] Okyay P, Ertug S, Gultekin B, Onen O, Beser E. Intestinal parasites prevalence and related factors in school children, a western city sample-Turkey. Bio Medical central Public Health. 2004; 4:64.

[2] Harhay MO, Horton J, Olliaro PL. Epidemiology and control of human gastrointestinal parasites in children. Expert Review of Anti-infective Therapy. 2010; 8: 219-234.

[3] Hsan-ul-Wadood A, Bari A, Ur RhmanQasim KF. Frequency of intestinal parasite infestation in children hospital quetta. Pakistan Journal of Medical Research. 2005; 44: 87-88.

[4] WHO. Amebiasis. Weekly Epidemiological Record. 1997; 72, 97-100.

[5] Haque R. Human intestinal parasites. Journal of Health, Population and Nutrition.2007; 25:387-391.

[6] Heidari A, Rokni MB. Prevalence of intestinal parasites among children in day-care centers in damghan-Iran. Iran. J. Pub. Health. 2003; 32: 31-34.

[7] Davis A, Cook C, Zumla A. Schistosomiasis: Manson's Tropical Diseases. London. Elsevier Science. 2003; 21:14311469.

[8] Mengistu Legesse, BerhanuErko. Prevalence of intestinal parasites among schoolchildren in a rural area close to the southeast of Lake Langano, Ethiopia.Ethiop.J.Health Dev. 2004; 18:116-120.

[9] Cauyan GA, Andaman DE, Maricar SM, et al. Prevalence of intestinal parasites among schoolchildren in a Coastal Rural Area of Maragondon, Cavite, Southern Luzon, Philippines. ActaManilana. 2008; 56:43-9.

[10] Woldemichael T, Endeshaw T, Shibre T, Gebre T, Haddis M, Tilahun $\mathrm{D}$, et al. Intestinal parasitic infections in western Abaya with special reference to Schistosomamansoni. Ethiopian Journal of Health Development. 1999; 13:25-26.
[11] Amare Mengistu, Solomon Gebre-Selassie, TesfayeKassa. Prevalence of intestinal parasitic infections among urban dwellers in southwest Ethiopia.Ethiop.J.Health Dev. 2007; 21:12-17.

[12] Tadesse Dejenie, Beyene Petros, Irrigation Practices and Intestinal Helminth Infections in Southern and Central Zones of Tigray.Ethiop.J.Health Dev. 2009; 23:48-56.

[13] Tadesse D, Tsehaye A. Impact of irrigation on the prevalence of intestinal parasite infections with emphasis on schistosomiasis in Hintallo-Wejerat, North Ethiopia. Ethiopian Journal of Health Science. 2008; 18:33-38.

[14] Merid Y, Hegazy M, Mekete G, Teklemariam S. Intestinal helminthic infection among children at Lake Awassa area, south Ethiopia. Ethiopian Journal of Health Development. 2001; 15:31-37.

[15] GirumTadesse. The prevalence of intestinal helminthic infections and associated risk factors among school children in Babile town, eastern Ethiopia. Ethiop.J.Health Dev. 2005; 19:140-147.

[16] World Health Organization. Basic Laboratory Methods in Medical Parasitology, Geneva, 1991.

[17] Monica Cheesbrough. District Laboratory Practice in Tropical countries. Part I, second edition, Cambridge University Press, 2009.

[18] Brooker S, Moulin A, Luoba D, Bundy M, Kremer D. Epidemiology of Single and Multiple Species of Helminth infections among School Children in Busia District, Kenya. East African Medical Journal. 2000; 77:157-168.

[19] Leykun J. Soil Transmitted Helminthic Infection and S. mansoni in School Children from Chilga District, North West Ethiopia. Ethiop J Health Science. 2001; 11:79-87.

[20] Abebe Alemu, Asmamaw Atnafu, Zelalem Addis, Yitayal Shiferaw, Takele Teklu, et.al. Soil transmitted helminths and schistosomamansoni infections among school children in zarima town, northwest Ethiopia. BMC Infectious Diseases. 2011; 11:189.

[21] Leykun J. Intestinal Helminthic Infection with Special Reference to S. mansoni in School Children in Adarkay District, North West Ethiopia. Ethiop J Health Dev.1997; 11:289-94.

[22] Tadesse Dejenie and Tsehaye Asmelash. Schistosomiasismansoni among School Children of Different Water Source Users in Tigray, Northern Ethiopia. MEJS. 2010; 2: 49-60.

[23] Basista R, Yoko O, Ramesh B, Basudha R, Keshav P, Chitra K, et al,. Gender variations in the prevalence of parasitic Infections and the level of awareness in adolescents in rural Nepal. Southeast Asian Journal of Tropical Medicine and Public Health.2001; 32: 575-580.

[24] Okpala H, Agwu I, Chimezie O, Nwobu G, Ohihoin A,. A survey of the prevalence of schistosomiasis among pupils in Apata and Laranto areas in Jos, Plateau State. Online Journal of Health Allied Sciences. 2004; 1:1. 